\title{
Germanica
}

\section{La génération de 68 et le passé des pères : entre accusation et identification inconsciente?}

Die 68er Generation und die Vergangenheit der Väter: zwischen Anklage und unbewusster Identifikation?

\section{Christine Schmider}

\section{(2) OpenEdition}

\section{Journals}

Édition électronique

URL : http://journals.openedition.org/germanica/511

DOI : 10.4000/germanica. 511

ISSN : 2107-0784

\section{Éditeur}

Université de Lille

\section{Édition imprimée}

Date de publication : 1 juin 2008

Pagination : 29-45

ISBN : 978-2-913857-21-6

ISSN : 0984-2632

\section{Référence électronique}

Christine Schmider, «La génération de 68 et le passé des pères : entre accusation et identification inconsciente? », Germanica [En ligne], 42 | 2008, mis en ligne le 01 juin 2010, consulté le 06 octobre 2020. URL : http://journals.openedition.org/germanica/511 ; DOI : https://doi.org/10.4000/germanica. 511

Ce document a été généré automatiquement le 6 octobre 2020.

(c) Tous droits réservés 


\title{
La génération de 68 et le passé des pères : entre accusation et identification inconsciente?
}

\author{
Die 68er Generation und die Vergangenheit der Väter: zwischen Anklage und \\ unbewusster Identifikation?
}

Christine Schmider

1 Dans le contexte de la Vergangenheitsbewältigung, les événements de 68 sont généralement considérés comme un moment de rupture important, marquant la fin d'une période de plus de vingt ans d'un refoulement collectif et individuel. La génération de 68 réclame des comptes à ses parents, refuse d'accepter leur silence sur le $\mathrm{III}^{\mathrm{e}}$ Reich et a tendance à condamner en bloc ses pères, incapables d'admettre leur compromission politique et morale. La littérature de l'époque se fait l'écho de cette relation intergénérationnelle très conflictuelle et met en scène des règlements de comptes familiaux. On s'accorde donc à dire que c'est, entre autres, grâce à l'insistance de la génération de 68 que la chape de plomb qui pesait sur le passé nazi en Allemagne commence à se fissurer. Toutefois, depuis un certain temps, le regard sur le rôle positif que les soixante-huitards ont joué dans la prise de conscience historique commence à changer. Non seulement on constate une nouvelle empathie de cette génération pour ses aînés, qui est également manifeste dans une littérature qui privilégie désormais les thèmes du pardon et de la compréhension. Mais de plus, des chercheurs comme le sociologue Harald Welzer mettent en doute la sincérité de la révolte des soixantehuitards contre leurs pères, la soupçonnant d'être, au fond, un désir d'identification inavoué, permettant de masquer, par le rejet, une tendance affirmative sous-jacente.

2 Thèse volontairement provocatrice? Opportunisme d'un chercheur qui joue sur l'actuelle remise en question des acquis de $68^{1}$ ? Ou regard lucide sur le mythe historique et mémoriel d'une jeunesse qui revendique d'avoir enfin affronté le passé de son pays? Afin de répondre à ces questions, nous nous interrogerons sur le rôle de la génération de 68 dans le contexte de la Vergangenheitsbewältigung, terme par lequel nous comprenons le travail mémoriel sur le passé, et nous analyserons sa remise en 
question politique, générationnelle et scientifique, ainsi que l'actuelle tendance à l'auto-critique de la part d'anciens soixante-huitards. Afin de mieux comprendre la relation complexe qu'entretient la deuxième génération d'après-guerre avec le $\mathrm{III}^{\mathrm{e}}$ Reich et ceux qui l'ont vécu, nous étudierons le roman d'un ancien acteur du mouvement étudiant, Stephan Wackwitz, qui, dans Ein unsichtbares Land (Un pays invisible) explore cette configuration familiale.

\section{Le poids du silence}

3 Si le discours mémoriel sur le passé nazi de l'Allemagne semble omniprésent aujourd'hui, cette hypermnésie ne doit pas faire oublier que le pays a longtemps refusé de faire face aux crimes du III ${ }^{e}$ Reich. Le climat politique de l'après-guerre, marqué par la reconstruction d'un pays économiquement et moralement anéanti, ainsi que la dénazification et la rééducation imposées par les Alliés ne sont guère propices au travail mémoriel. Dès 1949 se fait entendre la revendication du Schlussstrich. Cette incitation à tirer un trait sur le passé sera entendue par Konrad Adenauer qui, dans son discours inaugural à la chancellerie du 20 septembre 1949, souligne avec véhémence que le passé doit appartenir au passé ${ }^{2}$. Les premières lois d'amnistie seront adoptées en 1950 et la décennie sera marquée par le maintien ou la réintégration d'anciens fonctionnaires nazis dans les milieux administratifs, politiques ou universitaires. Ce phénomène, connu sous le terme de personelle Kontinuitäten contribue largement à la Geschichtsvergessenheit (l'amnésie historique) dont souffre la jeune République Fédérale. Le grand procès contre les bourreaux d'Auschwitz à Francfort entre 1962 et 1965 cristallise certes l'intérêt de l'opinion public, mais témoigne en même temps d'une restriction de la notion de culpabilité, dans la mesure où l'accusation ne s'adresse qu'aux personnes directement impliquées dans des crimes.

En diabolisant quelques coupables individuels, la société se dégage encore une fois de sa responsabilité historique et parvient à évacuer le débat sur le passé. Cette paralysie mémorielle qui provient de l'incapacité de porter le deuil (Die Unfähigkeit zu trauern) qu'Alexander et Margarete Mitscherlich ont diagnostiquée dans leur ouvrage du même nom, est quasi totale jusqu'à l'arrivée de cette génération ${ }^{3}$ que l'on nommera, aprèscoup, celle de $68^{4}$.

5 En refusant le silence qui pèse sur l'époque du III $^{\mathrm{e}}$ Reich et en demandant des explications aux pères qui ont participé à la guerre, la deuxième génération d'aprèsguerre fait exploser, avec une violence extrême, le consensus fondateur de la RFA qui repose sur la triade constituée par le miracle économique, la chape de plomb politique et l'adhésion au modèle démocratique par opportunisme plus que par conviction ( "Demokratie ohne Demokraten"5). Dans un contexte de contestation générale ${ }^{6}$, la révolte politique de la jeunesse de 68 est centrée sur une critique du fascisme, critique qui s'étend alors à tous les domaines, et notamment celui de la famille où elle surgit sous forme d'un conflit générationnel extrêmement dur. Une des manifestations les plus spectaculaires de cet antagonisme politico-familial est la Väterliteratur, cette littérature autobiographique des années 70 et 80 qui met en scène la rupture entre les parents supposés coupables d'aveuglement idéologique, de compromission morale et surtout d'un silence écrasant quant au passé, et leurs enfants qui les accusent, jugent et condamnent sans appel. Ces textes ${ }^{7}$ souvent surinvestis d'émotions violentes et d'affects contradictoires reflètent, par ailleurs, la situation discursive de l'époque. En 
s'inspirant de la Kulturkritik de l'École de Francfort, de la psychanalyse, des théories d' oral history et de la Nouvelle Subjectivité ${ }^{8}$, ils décrivent les répercussions politiques du silence et les séquelles psychologiques de la culpabilité inextricable des parents sur les enfants, et prônent un travail sur la mémoire comme seul moyen thérapeutique pour les individus et la société pétrifiée dans laquelle ils vivent.

Est-il pour autant justifié de dire que la découverte du passé, la mise en évidence des crimes nazis, la réflexion sur le travail de mémoire dans un pays dont l'identité reste à tout jamais marquée par le souvenir de la barbarie est l'exploit de cette génération ? Le but de notre travail n'est pas tant de juger du bien-fondé de cette vision, qui a longtemps été partagée par les historiens et l'opinion publique, mais d'analyser le retournement spectaculaire qui s'est opéré dans la perception, depuis le tournant du xxI ${ }^{\mathrm{e}}$ siècle.

\section{Mise en question de 1968 - bref rappel historique}

7 Les accusations violentes contre la génération de 68 ne sont pas nouvelles. C'est surtout sa posture de supériorité morale qui, dès le début, lui a été reprochée par ses aînés, murés dans la conviction que seuls ceux qui ont vécu le III $^{e}$ Reich pouvaient se permettre un jugement. La position morale radicale de la deuxième génération a d'autant plus été critiquée qu'elle s'opposait au consensus politique et public qui voulait que le passé soit tu dans l'intérêt supérieur d'une stabilisation de la démocratie allemande. À ce titre, la réaction du politologue Hermann Lübbe face à l'insistance de la génération de 68 à demander des explications à ses parents est tout à fait représentative. Dans son texte de 1983, Lübbe revendique la nécessité de taire des éléments biographiques compromettants ( kommunikatives Beschweigen [...] brauner Biographieanteile ») afin d'assurer l'adhésion au modèle démocratique ${ }^{9}$ et avance que l'attitude critique des jeunes vis-à-vis de la première génération induit une délégitimation du système politique de la RFA. Pour Lübbe, comme pour beaucoup de ses contemporains, le danger pour la démocratie ne vient pas de la persistance d'idées nazies ou de la présence sur la scène publique de personnalités au passé idéologique douteux, mais du refus d'empathie des jeunes vis-à-vis de leurs parents. Comme nous allons le voir dans l'analyse du débat actuel sur le rôle de 68, ce reproche adressé à la deuxième génération est réitéré aujourd'hui. Ce n'est pas la seule critique actuelle faite aux soixante-huitards, qui a déjà connu son heure de gloire au début des années 80 . Il en est de même avec l'accusation qui leur est faited'être responsable de la destruction des valeurs, traditions et autres bases de la société, comme le montre la diatribe de Ludolf Herrman :

Hitler haben wir, wenn auch vielleicht nicht endgültig, bewältigt. Nicht bewältigt haben wir die Bewältigung Hitlers, wie sie zur Studentenrebellion von 1968 und zu den fundamentalen Umwertungen der Folgezeit geführt hat. 50 Jahre nach der Machtergreifung ist Hitler für uns ein Gegenstand der Geschichte, unser Problem aber ist die Antwort auf ihn, wie sie in den Sechzigern gegeben wurde. Die Wende, die wir benötigen, besteht nicht darin, dass wir ein weiteres Mal 1933 oder 1945 verdauen, sondern dass wir den nachträglichen Ungehorsam gegen Hitler überwinden. [...] Die Rebellion von 1968 hat mehr Werte zerstört als das Dritte Reich. Sie zu überwältigen ist daher wichtiger, als ein weiteres Mal Hitler zu überwinden ${ }^{10}$. 

membre actif de la CDU et de l'Église catholique, s'explique, à notre avis, au moins autant par des raisons générationnelles que proprement politiques. Pour la comprendre, il faut impérativement tenir compte de l'importance de la constellation familiale comme vecteur privilégié de la transmission de mémoire et d'identité. « La révolte des étudiants contre la génération des pères [...] était un choc. Ce n'était pas le nationalsocialisme, mais le mouvement des étudiants [...] qui agissait comme véritable traumatisme pour la République Fédérale. D'où la rage qui en résulte ${ }^{11}$. Il nous semble alors que cette rage contre les idées de 68, après une période de latence historique, vient de ressurgir avec une nouvelle force depuis quelques années. Cela peut paraître d'autant plus étonnant que, depuis, les historiens ont pleinement confirmé la vision des soixante-huitards qui soupçonnaient la société allemande d'avoir fait une large place aux anciens nazis. Loin de relever de la paranoïa hystérique, ce jugement est désormais incontestable, tout comme, dans un retournement paradoxal, le rôle de cette génération dans le domaine de la Vergangenheitsbewältigung se trouve de plus en plus contesté $^{12}$.

\section{Les accusateurs accusés - le démontage de 68}

On peut distinguer quatre grands axes dans la tentative de déconstruction de l'image de 68 comme tournant positif dans l'histoire de la commémoration du passé nazi. Le premier est l'attaque idéologique menée dans les années 90 par une 'Nouvelle Droite' décomplexée qui, suite à l'écroulement du socialisme et à la réunification, entend réhabiliter certaines valeurs identitaires et conservatrices. Le deuxième obéit à une logique plus strictement générationnelle. Il provient d'une partie des enfants des soixante-huitards, ces jeunes, nés entre 1965 et 1975 , qui se reconnaissent dans la caractérisation de la "génération Golf" dont l'ouvrage du même nom de Florian Illies ${ }^{13}$ dresse le portrait et pour qui l'engagement anti-fasciste de leurs parents est totalement exagéré et anachronique. La troisième variante de la mise en question de 68 est celle d'une relecture critique et historique de ce mouvement faite par des historiens et sociologues. Elle repose sur un certain recul dû au temps et s'appuie sur l'avancement de la recherche qui permet un regard plus nuancé sur les exploits et les erreurs de ce mouvement. Enfin, le quatrième versant du démontage de 68 se présente sous la forme d'une auto-critique plus ou moins radicale qui revisite la rupture opérée avec les pères supposés nazis et questionne les motivations qui ont amené les jeunes à choisir des figures paternelles de substitution.

\section{L'attaque idéologique}

Le coup porté à l'héritage de 68 par la 'Nouvelle Droite' nous semble s'inscrire dans une conjoncture politique globale qui va dans le sens d'un anti-marxisme triomphant et d'un nationalisme affranchi. Les deux trouvent leur point culminant dans un ouvrage collectif à caractère programmatique publié en 1994 et intitulé Die selbstbewusste Nation $^{14}$. Ce recueil qui se veut un manifeste de l'intelligentsia néo-conservatrice accompagne l'essai extrêmement polémique de Botho Strauss Anschwellender Bocksgesang, dans lequel il dénonce le monopole culturel de la gauche, le libéralisme liberticide, l'ouverture gauchiste bien pensante qui met en danger l'identité nationale 
ainsi que toute la panoplie des idées d'une certaine gauche d'inspiration soixantehuitarde. Les autres contributions de l'ouvrage vont dans le même sens et fustigent « la haine de tout ce qui est allemand, semée par l'intelligentsia de gauche depuis $1968 »^{15}$ ou le fait qu' "après 68, la nation fut abandonnée sur la décharge de l'histoire (Kehrrichthaufen der Geschichte) et [...] que les valeurs familiales et religieuses se trouvèrent massacrées $»^{16}$.

\section{La rupture générationnelle}

11 La démolition du mythe de 68 engagée par des représentants de la 'génération Golf', si elle est infiniment moins virulente que celle des courants néo-conservateurs, nous semble tout aussi importante et peut-être, sous ses airs blasés et moqueurs, encore plus intégrale. Cette troisième génération d'après-guerre, que ses porte-parole autointronisés comme les auteurs regroupés sous le terme de Popliteratur ${ }^{17}$ définissent comme volontairement apolitique, hédoniste et consumériste (d'où le nom faisant allusion à la Golf prétendument indispensable à son bonheur) s'oppose résolument (mais sans passion, puisque les passions sont réservées aux choses importantes comme le fétichisme des marques) à l'héritage politique et social de ses parents. Son arme principale est l'ironie et sa cible privilégiée le discours dominant post-68 sur l'importance de l'histoire, l'engagement pour la Vergangenheitsbewältigung et la nécessité d'une conscience politique. Ce soi-disant monopole discursif dont la génération Golf dit avoir souffert tout au long de sa scolarité n'est pas attaqué pour des raisons idéologiques, mais parce qu'il parait, aux yeux de la troisième génération, foncièrement anachronique et - insulte suprême - ringard.

Das Verhältnis unserer Generation zur Geschichte allgemein und zum Holocaust ist dermaßen Roman-Herzoghaft unverkrampft, dass Kritiker dahinter Geschichtsvergessenheit vermuten, Ignoranz und Schlimmeres. Doch es ist eben das Problem der Generation der Gemeinschaftskundelehrer, dass sie bereits in der leidenschaftslosen Haltung, die die Generation Golf zur Geschichte einnimmt, Gefahren wittern, weil sie die Aufarbeitung der Vergangenheit noch mit soviel Leidenschaft gegen das Schweigen und den Widerstand ihrer Eltern durchsetzen mussten. Weil bei ihnen die Faschismusdebatte noch die gesamte Gesellschaft polarisierte ${ }^{18}$.

12 Ce professeur d'instruction civique dont les tentatives d'éveiller la conscience politique et historique de ses élèves sont, par ailleurs, décrites comme terriblement désuètes et obéissant au dogme du politiquement correct $^{19}$, incarne le soixante-huitard moralisateur que la génération Golf souhaite reléguer au passé, « denn die Abgrenzung gegen die Vorgängergeneration mit ihrer Moralhoheit war für uns früh eine entscheidende Lebensmaxime $»^{20}$. Forte de sa naissance tardive, la génération Golf fait de la Vergangenheitsbewältigung un objet scolaire et culturel gênant, non pas parce qu'elle éprouverait la moindre sympathie pour des idées nazies, mais en raison du changement de paradigme auquel elle aspire et qui remplacerait la morale par l'esthétique et la chose politique par le life-style.

\section{Le regard des chercheurs}

13 La recherche sur le rôle de 68 dans la Vergangenheitsbewältigung s'accorde aujourd'hui à dire que sa plus grande lucidité représente en même temps sa faiblesse historique. Nous parlons de la réflexion sur le fascisme qui se trouvait au cœur du mouvement et qui 
obligeait enfin la société allemande à s'interroger sur ses continuités politiques et personnelles. Toutefois, c'est cette même concentration sur le phénomène du fascisme et sa relation avec le capitalisme qui aboutit à une vision indifférenciée du danger fasciste, supposé avoir contaminé la démocratie allemande. Sous l'effet d'un durcissement de la critique politique, la distinction entre le régime hitlérien et le pouvoir de la bourgeoisie dans la démocratie parlementaire a tendance à disparaître, ce qui revient à minimiser le nazisme ${ }^{21}$. Le deuxième problème que pose la fixation sur le fascisme comme aboutissement inévitable d'une logique capitaliste réside dans l'aveuglement quant aux motivations idéologiques du nazisme, ayant mené au génocide du peuple juif ${ }^{22}$. La radicalisation du débat autour du fascisme ne rend pas pour autant plus visibles les victimes qui, de fait, se trouvent exclues de ce qui est devenu un conflit générationnel inter-allemand dont l'enjeu est un règlement de comptes entre parents et enfants et non pas le deuil envers les victimes.

Cette critique qui bénéficie certainement d'un recul temporel dont les représentants de la génération de 68 ne pouvaient, en toute logique, pas bénéficier, nous semble justifiée. Il s'agit d'une historicisation de l'affrontement entre parents et enfants qui ne met pas en cause le bénéfice que ce conflit a apporté pour la prise de conscience de la démocratie allemande. En revanche, la critique du sociologue Harald Welzer, spécialiste de la mémoire familiale et des relations inter-générationnelles, va beaucoup plus loin. En s'intéressant à la conjoncture actuelle que connaissent les romans familiaux ${ }^{23}$, il constate un retournement spectaculaire dans le regard que porte la deuxième génération sur l'implication de ses parents dans le régime hitlérien. Welzer constate ainsi que de nombreux romans actuels écrits par des soixante-huitards mettent en scène des dispositifs narratifs qui font de leur parents des «schuldlos Schuldige ${ }^{24}$ (des coupables innocents). Des auteurs comme Bernhard Schlink (Der Vorleser) et Ulla Hahn (Unscharfe Bilder) aspireraient alors à une réconciliation générationnelle, à un pardon rétrospectif qui atténuerait la position intransigeante dont ils ont fait preuve en 68 . Cette nouvelle empathie avec la $1^{\text {ère }}$ génération ne se réduit pas seulement aux romans évoquant l'époque nazie, mais se manifeste aussi dans la vague de publications, documentaires et téléfilms sur les victimes allemandes de la guerre, ainsi que dans la mémoire familiale ${ }^{25}$. Le sentiment d'avoir manqué de compassion ou au moins de compréhension à l'égard de ceux qui furent confrontés à la guerre et au régime hitlérien se fait alors entendre, avec force, au sein même de cette génération qui, en 68 , a rompu avec ses parents, en refusant en bloc leur modèle familial et politique. L'interprétation que donne Welzer de ce changement revient, de fait, à discréditer la position critique initiale qui paraît, à ses yeux, non comme une remise en question motivée par des convictions politiques, mais plutôt comme une tentative de renier toute ressemblance personnelle avec les parents. La nature extrêmement violente du réquisitoire que les enfants tenaient contre leurs parents s'expliquerait, selon lui, par leur volonté farouche de rejeter des affinités secrètes avec la génération de la guerre. La condamnation en bloc des pères cacherait, au fond, une projection non avouée qui repose sur des tendances identificatoires importantes.

Ce reproche n'est pas tout à fait nouveau. Déjà en 1977, dans leur postface à la $3^{\mathrm{e}}$ édition de leur ouvrage Die Unfähigkeit zu trauern, Alexander et Margarete Mitscherlich constataient que sous l'effet d'une radicalisation de la révolte, la jeunesse risquait de surinvestir des valeurs et des idées dogmatiques, et de glisser de plus en plus vers un fanatisme politique et moral. Et ils soulignaient le danger d'une régression de ces 
jeunes vers les comportements caractéristiques de leurs parents auxquels Hitler fournissait justement des valeurs univoques et incontestables ${ }^{26}$. Welzer reprend cette argumentation en soulignant à quel point l'orthodoxie des soixante-huitards, ainsi que leur fanatisme utopique les situent dans une continuité avec la génération parentale. L'investissement de la chose politique rapproche d'autant plus les soixante-huitards de la génération de la guerre que la vie politique dans la République Fédérale se caractérisait justement par sa nature dépassionnée et dépourvue d'attachement émotionnel. La disposition à l'endoctrinement et le pathos idéologique du mouvement étudiant renoueraient alors avec ceux dont la première génération a fait preuve. Par ailleurs, l'argument des pères prétendant que seul le fait d'avoir vécu leur époque historique permettrait un jugement sur leur comportement est repris, comme le fait remarquer Welzer, à l'identique dans le discours d'anciens soixante-huitards justifiant les actions violentes lors de la révolte. Et finalement, le sociologue voit dans les émeutes de 68 une mise en scène inconsciente des fantasmes martiaux de la jeune génération. Dans l'impossibilité de connaître les combats, l'héroïsme et la camaraderie que leurs aînés ont vécus lors de la guerre, leurs enfants auraient cherché à suppléer ces expériences par leur révolte, dirigée justement contre le modèle de leurs parents.

Il nous semble que les remarques de Welzer sur la constellation complexe qui réunit les soixante-huitards et leurs parents, même s'il se complaît quelque peu dans sa pose provocatrice, ne manquent pas de pertinence. Cela dit, si ses critiques apportent des éléments importants à l'analyse de l'histoire générationnelle, elles n'amenuisent pas pour autant le rôle de 68. Par rapport à tout événement historique significatif, la deuxième génération se trouve, par la force des choses, face à une double exigence historique et générationnelle: juger du rôle de ses parents dans le passé et se situer dans une continuité de transmission et de filiation. Dans le cas du III Reich et de la Shoah, il est évident que cette double exigence est inconciliable. L'inscription dans une histoire générationnelle assumée ne peut que se heurter au refus des jeunes d'accepter un tel héritage, refus d'autant plus violent qu'il est forcément marqué par la peur de porter, en soi, les germes de ce qui a rendu possible, chez les parents, le basculement dans la barbarie. Face à cette contradiction, il est, à nos yeux, tout à fait logique que l'attitude des jeunes de 68 envers leurs parents soit foncièrement violente et ambiguë. Mais l'ambiguïté de ces prises de position, n'invalide, à notre sens, ni le constat initial les pères se sont murés dans leur refus de parler à leurs enfants, faisant ainsi du passé un tabou -, ni les conséquences de leur révolte - le silence fut rompu grâce à l'insistance des enfants. Il nous semble alors que l'argument qui prétend que la motivation des jeunes fut moins pure que ce qu'ils ont voulu croire ne disqualifie pas en soi la rupture que leur révolte a constituée pour la conscience politique et mémorielle de l'Allemagne.

\section{L'auto-critique des soixante-huitards}

17 De toutes les mises en question des idées de 68 , la plus surprenante est peut-être celle des anciens soixante-huitards eux-mêmes. Qu'il s'agisse d'hommes politiques, de journalistes ou d'écrivains, nombreux sont ceux qui actuellement, prennent leur distance avec le mouvement, font leur mea culpa public quant à leur implication ou portent un regard pour le moins ironique sur leur engagement antérieur ${ }^{27}$. Nous ne pouvons bien évidemment pas exclure que dans certains cas, cette démarche soit motivée par un opportunisme politique. Mais en ce qui concerne la position des 
soixante-huitards par rapport au passé et à la pratique mémorielle, l'auto-critique nous semble relever d'un recul critique et d'une réflexion assez nuancés. L'article de Christian Schneider sur « L'holocauste comme objet générationnel » est, à ce titre, tout à fait significatif. Il part du constat que la génération de 68 monopolise le domaine de la Vergangenheitsbewältigung qu'elle considère comme son apanage, de façon à exclure la troisième génération du débat mémoriel. Une telle monopolisation s'expliquerait par le sentiment d'être la première génération à avoir pleinement compris et accepté la signification de la Shoah, la première à avoir ressenti l'empathie et le deuil pour les victimes, à avoir fait remonter à la surface un pan de l'histoire jusque-là occulté. Cette singularité de la Shoah qu'ils supposent être les premiers, en Allemagne, à avoir perçue, leur confère en même temps une identité générationnelle singulière. Fruit d'un combat contre les parents, ils la défendent aussi contre leurs propres enfants, leur reprochant en quelque sorte d'être incapables d'une empathie avec les victimes à la hauteur de la leur.

Un deuxième aspect qui contribue également au fait que cette génération se considère comme les gardiens de la Vergangenheitsbewältigung concerne leur identification aveugle avec des pères de substitut. Ce phénomène dont fait également mention le si bien nommé " roman familial » de Wackwitz se manifeste dans l'importance que les philosophes de l'École de Francfort ont prise pour les jeunes de $68^{28}$. En tant qu'intellectuels juifs qui ont dû fuir l'Allemagne nazie, ils représentent, pour de nombreux jeunes, des figures paternelles alternatives, représentant une autre histoire de l'Allemagne et la possibilité de s'inscrire dans une généalogie valorisée ${ }^{29}$. Une telle filiation fantasmée qui illustre à la perfection la constellation décrite par Freud dans «Der Familienroman des Neurotikers » exclut forcément la génération suivante qui se trouve reléguée dans une position d'observateur de l'histoire.

\section{Un pays invisible}

Le roman de Stephan Wackwitz apporte une contribution tout à fait intéressante au débat sur le rôle de 68 dans le contexte de la Vergangenheitsbewältigung. Il compte parmi les prises de position les plus nuancées des anciens acteurs de 68 et porte un regard lucide sur les erreurs politiques et personnelles qui ont marqué l'époque, mais aussi sur la nécessité historique de ce mouvement.

\section{Le passé fantomatique}

En explorant les traces de son passé familial, Stephan Wackwitz (né en 1952) met en perspective les biographies de trois générations d'hommes, le grand-père du narrateur, son père et lui-même, tout en les inscrivant dans le passé de son pays. Afin de reconstituer l'histoire familiale et l'histoire politique dans leur enchevêtrement inextricable, il entreprend un voyage mémoriel à travers les mémoires de son grandpère qui entre 1921 et 1933 fut pasteur pour la communauté allemande près d'Auschwitz, avant d'officier en Namibie d'où il revint en Allemagne en 1939. Cette lecture incite le narrateur à partir à la recherche de la «contrée fantomatique qui s'étend entre la Vistule et la Sola, entre les Carpates et les marais, entre Kattowitz et Auschwitz. [...] (dans ce paysage auquel ma famille mais semble-t-il tout le pays aussi sont attachés depuis un demi-siècle de manière étrange et secrète)»(p.65). Pour 
Wackwitz, le but de ce voyage est double : mieux comprendre «la prostration » (p. 23), le mutisme obstiné et les accès de bouderie et d'abattement de ce grand-père qui, déjà de son vivant, était "quelqu'un dont le discours nécessitait des explications historiques» (p. 27), mais aussi lever le secret de son «propre non-avenir» (p. 64), de sa mollasserie de jeune homme, de cet « état très particulier, gênant et fort désagréable à mon goût, qui était fait d'un mélange de paralysie et de subordination résignée, mais en même temps d'insuffisance, de rêverie, de honte et de révolte réprimée » (p. 61).

21 Au fil de la lecture du roman, on comprend que l'absence de perspective dont souffre le narrateur provient du fait que le présent est hanté par le passé et par les non-dits qui s'y rapportent. Son histoire est phagocytée par la proximité géographique et temporelle du lieu mythique familial - le presbytère d'Anhalt où rôdait le fantôme d'un ancien pasteur, où officiait son grand-père et où naquit son père - avec cet autre lieu mythique, Auschwitz, nom qui « qualifie pour nous un trou noir de l'histoire du monde moderne, un trou où tombe tout ce qui l'approche et dont les rebords forment un horizon menaçant qui cerne tout ce qu'on pourra dire dorénavant sur le siècle dernier et sur l'Histoire en général» (p.153). Jamais personne dans sa famille n'avait fait remarquer que ces deux endroits «n'étaient distants que d'une longue promenade ou d'une petite décennie à peine » (p.11), et ce n'est qu'en se rendant dans l'ancienne demeure familiale que le narrateur s'en rend compte, tout comme il s'aperçoit que le fantôme du presbytère d'Anhalt, en d'autres mots, la persistance du passé dans le présent, «avait continué de hanter ma vie » (p.12). Afin de chasser ce sortilège qu'il met sur le compte de l'incapacité du grand-père à communiquer avec sa famille, le narrateur s'intéresse, pour la première fois, après "des décennies de silence et d'indifférence nerveuse » (p.38) à ce personnage tant méprisé. Se profilent alors certaines affinités et ressemblances inattendues entre son grand-père et lui-même.

Il existe des parallèles plus importants que notre goût commun à fumer le cigare et exercer notre profession à l'étranger. Les petites ressemblances apparemment fortuites ainsi que les plus importantes, qui ne peuvent être des hasards, se sont mises en place malgré ma volonté. Au cours de ma lecture, j'ai parfois eu l'impression qu'au fil des décennies ma vie et celle de mon grand-père s'étaient accordées dans mon dos. Il m'a fallu presque un demi-siècle [...] pour déceler ces ressemblances. Ce furent des retrouvailles émouvantes, mais pas seulement réjouissantes (p. 38-39).

Ces affinités révèlent une continuité transgénérationnelle dans les comportements que le narrateur s'était toujours efforcé de nier et qui créent une constellation familiale et historique dans laquelle la jeunesse de 68 apparaît comme l'héritière de la génération de la guerre, malgré sa volonté farouche de s'y opposer et de s'ériger en contre-modèle.

\section{Rêves révolutionnaires et égarements idéologiques - le retour du passé}

C'est seulement après la mort du grand-père que le narrateur voit se profiler quelques ressemblances insoupçonnées, notamment en ce qui concerne leur fascination commune pour les grandes ruptures historiques. En lisant le Journal de son grand-père, il tombe sur un passage consacré «à la fin du monde survenue en 1918, à l'esprit allemand, à la substance populaire et à la révolution» (p. 116), en somme, à toutes ces idées « étrangement embrouillées et national-autistiques » qu'il exècre tant, mais dans lesquelles il est obligé de reconnaître, en filigrane, sa propre exaltation 
révolutionnaire, les rêves de changements radicaux qui habitent sa génération et comme " un petit fantôme aux pâles contours, mes propres égarements politiques à l'âge de vingt-quatre ans » (p. 115).

Ces égarements du jeune Wackwitz ont une double origine : le silence des pères, en l'occurrence du grand-père qui oppose une fin de non-recevoir aux questions du petitfils concernant son éventuelle culpabilité, en conjurant ainsi les fantômes du passé qui vont continuer à poursuivre les générations suivantes et - comme réaction à ce silence - la tentative désespérée du narrateur et de sa génération de rompre à tout prix avec les parents et grands-parents ayant fait la guerre. «J'allais vainement essayer de tout faire autrement, même si j'étais loin de m'en douter, ce matin-là. J'allais gâcher une grande partie de mes précieuses années d'études en adhérant à une ligue communiste carnavalesque - une servitude volontaire que je me suis infligée en jetant par-dessus bord et le vaste monde et la liberté » (p. 64). Plutôt que d'échapper à l'endoctrinement qu'a subi la génération de son grand-père et de son père, le narrateur est donc condamné, comme obéissant à une compulsion de répétition, à connaître à son tour l'aveuglement idéologique. Ainsi, en 1974, lors d'un voyage en RDA, et en tant que membre de la ligue d'étudiants marxiste MSB Spartakus, il se laisse berner par l'image que le socialisme est-allemand souhaite donner de lui-même :

À cet égard, je n'ai sans doute jamais été aussi proche de mon grand-père qu'en 1975 [...] et plus généralement durant les années où j'ai voulu m'éloigner le plus possible de lui et de ses convictions; c'est-à-dire à l'époque de mon flirt avec cet autre totalitarisme, que je me suis infligé à des fins provocatrices (et à mes dépens). C'est pourquoi je suis sans doute le moins habilité de nous tous à reprocher à mon grand-père son aveuglement de Luckenwalde (p. 260-261).

Le livre de Wackwitz décrit, avec une rare acuité, comment le passé refoulé du pays engendre des revenants qui hantent la jeunesse de 68 . Sous l'influence spectrale du passé, cette génération pense devoir continuer le combat perdu, voire abandonné d'avance par leurs parents contre le fascisme, en tombant, toutefois, dans un nouvel aveuglement idéologique. En témoignent l'identification presque hystérique avec les victimes du nazisme, tout comme la conviction d'avoir affaire, avec la République Fédérale, à un nouvel État fasciste. Le personnage de Rudi Dutschke est, à ce titre, représentatif de toute une génération. "C'est à cette époque qu'a commencé pour Rudi (et ensuite pour tous les autres) l'identification théâtrale avec les victimes, notre espèce de jeu chamanique qui consistait à se transformer en Juifs et en communistes " (p. 298). Car Dutschke exprime, dans son propre corps, dans sa propre voix, cette identification avec les morts du passé, avec les victimes de la barbarie en s'inscrivant dans une filiation fantomatique :

La voix de Rudi Dutschke [...] ne semble pas dater de la fin des années soixante mais des années vingt. Ses voyelles singulièrement allongées et son $\mathrm{R}$ roulé rappellent les intonations d'un orateur étranger d'avant la guerre ou de l'entre-deux-guerres qui aurait appris l'allemand tardivement, mais parfaitement (d'un professeur judéoroumain qui aurait passé presque tout sa vie à New York [...]). La voix de Dutschke semble avoir été enregistrée lors d'une réunion de spiritisme. Alors qu'il était encore en vie, Dutschke avait l'air de parler le langage des morts. [...] On aurait dit que cet homme, alors très jeune encore, se concevait, au sens corporel du terme, comme le médium de théories oubliées dont les inventeurs et les orateurs originaux avaient été assassinés dans les camps de concentration (p. 286-287). 
projection identificatrice, ainsi que le glissement temporel qu'elle implique caractérisent toute une génération, et le narrateur la vit à son tour lors d'une manifestation à laquelle il participe en 1974 à Tübingen :

La nuit tombe [...]. La bande magnétique diffuse la voix de Ernst Bloch sur la place ou l'allée. [...] . Dès que sa voix avait retenti, ma nouvelle veste en cuir et moi-même nous étions en tout cas retrouvés dans une autre époque, dans un pays rempli de fantômes. Goebbels, Hitler et Göring n'ont pas encore remporté la victoire à Berlin. Walter Benjamin rencontre au café le possesseur de cette voix qui résonnait sur la place en 1976 [...] Mon grand-père marchera bientôt sous les tilleuls, à la lueur des flambeaux, sous les signes de la main d'un Hindenburg lignifié, presque momifié déjà, il va [...] ressusciter en Adolf Hitler ses rêves d'avant-guerre. Mes camarades seront bientôt battus, à mort pour certains, dans les caves et les prisons improvisées de la SA. [...]. Je lutte à mon époque contre Goebbels, la SA et mon grand-père (p. 294-295).

Des années plus tard, le narrateur commente ce chavirement temporel et la surexcitation idéologique qui l'accompagne :

Il me restait encore beaucoup d'années pour réfléchir à la confusion des temps qui nous était tombée dessus, à nous tous, dans la décennie d'après 1968 [...]. Durant toutes ces années de réflexion, le jeu des méprises et des apparitions qui frisait alors la folie m'a semblé de plus en plus clairement être un remake de l'Hamlet de Shakespeare - modèle littéraire auquel se référaient les prouesses remuantes et gesticulantes, les mises en scène hystériques, les grossesses nerveuses historiques, les méconnaissances de soi et les illusions sensorielles dont nous étions prisonniers - et aujourd'hui, après la rédaction de ce récit sur mon grand-père, il me semble par ailleurs, que la prophétie hamletienne de 1968, c'est-à-dire notre métamorphose à tous en personnages de cette tragédie, avait dû commencer à Luckenwalde entre 1940 et 1945 (p. 296).

Cette dernière remarque de Wackwitz nous permet de dire que son roman familial, pour autant que l'auteur semble regretter son passé de soixante-huitard comme égarement idéologique, comme gâchis d'énergie et comme illusion lui ayant coûté cher en temps et en bonheur de vivre, ne peut pas être assimilé à une mise en cause historique de 68. Le cheminement politique et personnel du narrateur aboutit bien au constat que «ma vie était devenue très semblable à celle d'un homme auquel, lorsqu'il vivait encore et que j'étais jeune, je ne voulais ressembler pour rien au monde » (p. 38), battant ainsi en brèche l'opposition radicale entre la génération de la guerre et les jeunes du mouvement étudiant qui fut si constitutive de 68. Or, le repositionnement au sein de la configuration familiale et l'apaisement générationnel que vit le narrateur d' Un pays invisible, à l'image de toute une génération, n'implique aucunement un aveuglement face aux compromissions politiques des parents. Et si le roman se fait bien l'écho d'un certain nombre de reproches contre une époque qui, encore aujourd'hui, polarise le débat politique, il ne se prête jamais à une récupération "révisionniste" qui ferait de 68 un accident regrettable de l'histoire. Car pour Wackwitz, 68 reste, avant tout une tentative désespérée de conjurer les revenants de l'histoire allemande, « une réponse tardive au fait que mon grand-père avait vécu dans un pays réellement hanté par des fantômes » (p. 279). 


\section{NOTES}

1. Cf. la mise en question du ministre des Affaires étrangères de l'époque, Joschka Fischer, lors d'un débat au Bundestag en janvier 2001. Fischer fut notamment attaqué par l'opposition en raison de sa participation à la révolte des étudiants et de son passé de soixante-huitard.

2. Klaus Stüwe : Die Rede des Kanzlers. Regierungserklärungen von Adenauer bis Schröder, Wiesbaden, Verlag für Sozialwissenschaften, 2005.

3. La notion de génération nécessite quelques clarifications préalables. Tout d'abord, nous utilisons ce terme dans le sens que lui a donné Karl Mannheim dans «Das Problem der Generationen », in: Wissenssoziologie, Neuwied, Luchterhand, 1964, p. 509-565. Mannheim comprend une génération non pas comme un ensemble de personnes réunies par le hasard d'une naissance au même moment. Elle se caractériserait plutôt par un vécu similaire, basé sur un événement marquant partagé. Par conséquent, l'appartenance à une même génération relève du culturel, plutôt que du temporel. Les recherches récentes en psychologie historique ont, par ailleurs, permis d'enrichir la notion de génération d'une dimension diachronique. (Cf. Christian Schneider: "Noch einmal 'Geschichte und Psychologie'. Generationengeschichte als Modell psychohistorischer Forschung ", in : Mittelweg 36, 1997, Heft 1 et 2). Nous estimons alors que lors du processus générationnel, certaines configurations inconscientes comme des peurs ou des attentes se trouvent transmises d'une génération à l'autre.

4. À ce propos, il faut rappeler qu'en Allemagne, la notion de génération, qui a connu un formidable écho depuis les années 80, a été peu utilisée dans les années 60 et 70, et encore moins pour désigner les jeunes que l'on qualifie, à l'époque, de Protest- ou Studentenbewegung ou encore d'APO.

5. Cf. Birgit Schelling: «Der Wandel von Einstellungen und Mentalitäten nach dem Übergang vom Nationalsozialismus zur Bundesrepublik », in : W. Bergem (éd.) : Die NS-Diktatur im deutschen Erinnerungsdiskurs, Opladen, Leske+Budrich, 2003, p. 41-58 ; Julia Kölsch : Politik und Gedächtnis. Zur Soziologie funktionaler Kultivierung von Erinnerung, Wiesbaden, Westdeutscher Verlag, 2000.

6. La commémoration de 68 battant son plein, les publications sur cette période historique sont innombrables. Gerd Koenen, ancien soixante-huitard et auteur de l'ouvrage autocritique très remarqué Das rote Jahrzehnt. Unsere kleine deutsche Kulturrevolution 1967-1977, Francfort/M, Fischer, 2006 qui a notamment inspiré Stephan Wackwitz, a établi une bibliographie raisonnée d'environ 1000 titres qui est consultable sur internet (www.gerd-koenen.de).

7. Nous n'énumérons que quelques-uns des ouvrages les plus connus : Peter Henisch : Die kleine Figur meines Vaters (1975), Elisabeth Plessen : Mitteilungen an den Adel (1976), Ruth Rebmann : Der Mann auf der Kanzel (1979), Peter Härtling : Nachgetragene Liebe (1980), Christoph Meckel : Suchbild : Über meinen Vater (1980), Günter Seuren : Abschied von einem Mörder (1981).

8. Cornelia Blasberg : «Erinnern? Tradieren? Erfinden? Zur Konstruktion von Vergangenheit in der aktuellen Literatur der dritten Generation ", in : C. Blasberg, J. Birkmeyer (éd.) : Erinnern des Holocaust? Eine neue Generation sucht Antworten, Bielefeld, Aisthesis, 2006, S. 167-168.

9. Hermann Lübbe: "Der Nationalsozialismus im deutschen Nachkriegsbewusstsein », in : Historische Zeitschrift, 1983, 236, p. 579-585.

10. Ludolf Herrman: "Hitler, Bonn und die Wende. Wie die Bundesrepublik ihre Lebenskraft zurückgewinnen kann », in : Die politische Meinung, 1983, 204, p. 13.

11. Hajo Funke: Von der Gnade der geschenkten Nation. Zur politischen Moral der Bonner Republik, Berlin, Rotbuch Verlag, 1988, p. 15.

12. Cf. Gerd Steffens : «Im Generationen-Container. Die 68er und ihre Nachfolger », in : Blätter für deutsche und internationale Politik, 2001, $\mathrm{n}^{\circ}$ 6, p. 737-746.

13. Florian Illies : Generation Golf. Eine Introspektion, Berlin, Argon, 2000. 
14. Heimo Schwilk, Ulricht Schacht (éd.) : Die selbstbewusste Nation, Berlin, Francfort, Ullstein, 1994.

15. Gerd Bergfleht : «Erde und Heimat. Über das Ende der Ära des Unheils », in : Ibid., p. 104.

16. Ansgar Graw : «Dekadenz und Kampf. Über den Irrtum der Gewaltlosigkeit », in : Ibid., p. 282. Pour une analyse approfondie de la rhétorique néo-conservatrice de cet ouvrage, cf.: Julia Kölsch : op. cit., p. 159-174. Cf. également les articles de Reinhard Mohr : «Zorn auf die roten Jahre ", in: Der Spiegel, 2001/4, 22, p. 192-195 et de Jochen Bölsche : "Die verlorene Ehre der APO » in: Der Spiegel, 2001/29 janvier, p. 68 -85 qui mettent en lumière la tendance néoconservatrice actuelle d'attribuer la perte des valeurs à 68 .

17. Il s'agit du groupe autour de Christian Kracht, Benjamin Stuckrad-Barre, Wolf Haas, Andreas Mand.

18. Florian Illies: op. cit., p. 174.

19. Ibid., p. 100.

20. Ibid.

21. Wolfgang Bergem : «Barbarei als Sinnstifung ?», in : W. Bergem (éd.) : op. cit., p. 92.

22. Hans-Ulrich Thamer : "Der Holocaust in der deutschen Erinnerungskultur vor und nach 1989 », in : C. Blasberg, J. Birkmeyer (éd.) : op. cit., p. 81-94.

23. Christine Schmider: «Écriture et réécriture de la mémoire allemande : le nouveau roman familial », in : C. Hähnel-Mesnard, M. Liénard-Yétérian et C. Marinas (éd.) : Culture et mémoire. Représentations contemporaines dans les espaces mémoriels, les arts visuels, la littérature et le théâtre, Palaiseau, Éditions de l'École Polytechnique, 2008.

24. Harald Welzer: "Schön unscharf. Über die Konjunktur der Familien- und Generationenromane ", in : Literatur. Beilage zum Mittelweg 36, 2004, n 1, p. 53-64.

25. Christine Schmider : «La mémoire en question - Unscharfe Bilder d'Ulla Hahn », in: La mémoire historique : interroger, construire, transmettre, Presses de l'Université d'Angers, 2006, p. 165-172.

26. Alexander et Margarete Mitscherlich: Die Unfähigkeit zu trauern. Grundlagen kollektiven Verhaltens, München, Piper, 1967, p. 368.

27. Cf. Gerd Koenen: op. cit.De nombreux auteurs littéraires comme Friedrich Christian Delius, Peter Schneider, Uwe Timm, Ulrike Kolb ou Elke Schmitter proposent une réflexion critique sur le décalage entre idéal politique et vie personnelle et remettent en cause, après-coup, la condamnation sans appel de leurs parents par les enfants qu'ils étaient.

28. Chez Wackwitz, c'est Jürgen Habermas qui, dans le chapitre "Cinq professeurs, rêves de Jürgen Habermas » figure comme modèle intellectuel et substitut paternel. "J'avais été fier de Jürgen Habermas [...]. J'avais découvert un des nôtres. Voilà quelqu'un qui allait m'aider contre mon grand-père » (Stephan Wackwitz, Un pays invisible. Roman familial, Paris, Éditions L. Teper, 2007, p. 204. Toutes nos citations sont issues de cette édition). Avec le recul, cette structure d'identification est décrite, par l'auteur, comme symptôme d'un esprit divaguant, comme 'syndrome d'Hamlet'. "Car si Bloch et Lukács, Walter Benjamin, Léviné, Trotski et Karl Liebknecht ont été nos rois et nos pères, nous étions nous-mêmes des princes danois » (p. 298).

29. Le diagnostic qui voit dans la génération de 68 des enfants à la recherche de figures paternelles susceptibles de remplacer des pères réels, dépourvus d'autorité morale ou parentale, est partagé par de nombreux détracteurs de ce mouvement. Ainsi, Sophie Dannenberg (née en 1971), dans son roman Das bleiche Herz der Revolution (2004), dresse un portrait au vitriol de ces jeunes qui, ayant grandi sans pères ou avec des pères brisés par la guerre et la captivité, se vouent à des figures paternelles autoritaires comme Stalin, Mao ou Che Guevara. Elle voit, dans cette adhésion aux pères totalitaires, la même recherche d'un père imaginaire tout-puissant qu'à l'époque hitlérienne. Le roman de Dannenberg est un règlement de compte avec les parents soixante-huitards qui reprend la quasi-totalité des arguments que nous avons énumérés dans nos différents sous-chapitres. Elle reproche ainsi à la génération de 68 son manque d'empathie pour ses parents qu'elle explique dans un revirement provocateur non pas par le fait que ceux-ci aient 
mené une guerre d'agression, mais qu'ils l'aient perdue (Sophie Dannenberg: Das bleiche Herz der Revolution, Berlin, BTV, 2006, p.303). Elle dénonce l'indifférence violente de l'éducation antiautoritaire que les parents, en 68 , ont fait subir à leurs enfants et les accuse, en parfaite représentante de cette génération Golf évoquée par Illies, d'endoctrinement anti-fasciste primaire (p. 238).

\section{RÉSUMÉS}

Dans le contexte de la Vergangenheitsbewältigung, les événements de 68 sont généralement considérés comme un moment de rupture important, marquant la fin d'une longue période de refoulement collectif et individuel. On s'accorde à dire que c'est, entre autres, grâce à l'insistance de la génération de 68 que la chape de plomb qui pesait sur le passé nazi de l'Allemagne commence à se fissurer. Toutefois, depuis un certain temps, le regard sur le rôle que les soixantehuitards ont joué dans la prise de conscience historique commence à changer. La sincérité de leur révolte, soupçonnée de relever d'un désir d'identification inavoué et d'un rejet d'affinités sousjacentes, est contestée. Ces remises en question politique, générationnelle et scientifique ainsi que l'actuelle tendance à l'auto-critique de la part d'anciens soixante-huitards sont analysées dans notre contribution. Dans ce contexte, l'étude du roman Ein unsichtbares Land (Un pays invisible) d'un ancien acteur du mouvement étudiant, Stephan Wackwitz, permet de mieux comprendre la relation complexe qu'entretient la deuxième génération d'après-guerre avec le III ${ }^{\mathrm{e}}$ Reich et ceux qui l'ont vécu.

Die Ereignisse von 68, die im Kontext der Vergangenheitsbewältigung üblicherweise als ein entscheidender Bruch wahrgenommen werden, bedeuten das Ende einer langen Periode kollektiver und individueller Verdrängung. Es ist, so die allgemeine Annahme, zu einem nicht unbedeutenden Teil dem Insistieren der 68er zu verdanken, dass das bleierne Schweigen, das auf der nationalsozialistischen Vergangenheit Deutschlands lastete, gebrochen wurde. Seit einiger Zeit scheint sich jedoch die Einschätzung der 68er und ihrer Einflussnahme auf das historische Bewusstsein zu ändern. Die Authentizität ihrer Revolte, die im Verdacht steht, einer unbewussten Identifikation und der Abwehr unterschwelliger Affinitäten zu gehorchen, wird in Frage gestellt. Diese politische, generationsbedingte oder auch wissenschaftliche Hinterfragung sowie die aktuelle Tendenz zur Selbstkritik seitens ehemaliger 68er werden im vorliegenden Beitrag untersucht. Literarisch ausgeführt wird letztere im Roman Ein unsichtbares Land von Stephan Wackwitz, einem ehemaligen Vertreter der Studentenbewegung, der das komplexe Verhältnis der 68er zum Dritten Reich und zur Kriegsgeneration beleuchtet.

\section{INDEX}

Mots-clés : génération des pères, 1968 
AUTEUR

\section{CHRISTINE SCHMIDER}

Université de Nice-Sophia-Antipolis/CRHI 